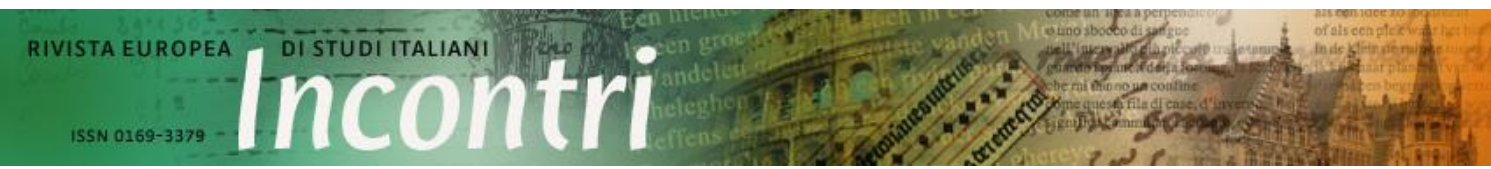

Anno 35, 2020 / Fascicolo 2 / p. 7-9 - www.rivista-incontri.nl - http://doi.org/10.18352/incontri.10348 (c) The author(s) - Content is licensed under a Creative Commons Attribution 3.0 Unported License Publisher: Werkgroep Italië Studies, supported by Utrecht University Library Open Access Journals

\title{
Religioni letterarie, una via alternativa al contemporaneo?
}

\section{Marco Zonch}

A distanza di quasi quindici anni dalla pubblicazione di Gomorra (2006), e a dodici da quella di Allegoria 57 (2008), il dibattito attorno alle trasformazioni avvenute a cavallo tra vecchio e nuovo millennio non si è ancora esaurito. Infatti, benché un accordo sia stato sostanzialmente raggiunto - il cambiamento sarebbe cioè da leggersi come un "ritorno" a realtà/verità e impegno - resta ancora da stabilire come ciò sia stato possibile dopo il postmodern(ism)o e la French Theory. Che una lettura definitiva di questo passaggio manchi risulta del resto evidente quando si prendano in considerazione i continui ritorni all'io so, la comparsa di sempre nuove proposte e categorie interpretative, e l'imbarazzo che sembra accompagnare l'uso di una di queste, impegno, percepita come inadeguata ma per cui non sembrano essere disponibili alternative. Insomma, senza troppo sbagliare potremmo dire che realismo, testimonianza, fiction, non-fiction, impegno ecc. si siano fin qui comportate come la proverbiale coperta che, troppo corta, lascia i piedi freddi.

Le difficoltà in cui si è imbattuta la critica possono forse trovare spiegazione nel fatto che, a dispetto delle differenze, tutti $i$ tentativi fin qui condotti abbiano affrontato il problema in termini esclusivamente epistemologici. ${ }^{1}$ Lasciando per ora da parte la questione dell'impegno, si può cioè affermare che a opere come Gomorra o Campo del sangue (1997), per fare un altro esempio, sia stato "soltanto" chiesto per quali ragioni e con quali modalità sia stato per loro possibile far di nuovo corrispondere parole e cose, senza che le prime si trasformassero 'in una polvere di finzioni', ${ }^{2}$ e anzi in alcuni casi riuscendo perfino ad attribuir loro valore di verità. Questo tipo di approccio, è forse importante sottolinearlo, è stata del resto adottata anche da chi come Arturo Mazzarella ha negato legittimità alle pretese di questi testi. ${ }^{3} \mathrm{E}$ se sembra dunque impossibile reperire esempi di approcci differenti, il dubbio che proprio allo sguardo epistemologico vadano imputate alcune delle difficoltà critiche sembra essere, se non altro, legittimo.

\footnotetext{
1 'Il problema', scrive per esempio Palumbo Mosca nel suo L'invenzione del vero (2014), 'non è quello di recidere il legame tra letteratura e realtà, bensi di adeguare la forma a una diversa epistemologia che, per tutto il corso del secolo, è andata sottraendo certezze al soggetto conoscente.' (R. Palumbo Mosca, L'invenzione del vero, Roma, Gaffi, 2014, p. 45). Ma anche Gianluigi Simonetti appoggia le pagine della "sua" letteratura circostante dedicate al problema della realtà a quanto ne dicono, appunto, 'gli epistemologi' (G. Simonetti, La letteratura circostante. Narrativa e poesia nell'Italia contemporanea, Bologna, Il Mulino, 2018, p. 45), pur sperando 'nel futuro di una narrativa che accolga, nella sua idea di realtà, tanto la materia più buia e reificata quanto la tentazione della luce, la nostalgia dell'assoluto' (ivi, p. 268). La citazione interna è di W. Siti, Il realismo è l'impossibile, Roma, Nottetempo, 2013, p. 59. 2 R. Donnarumma, Ipermodernità. Dove va la narrativa contemporanea, Bologna, Il Mulino, 2014, p. 12.

${ }^{3}$ Cfr. A. Mazzarella, Politiche dell'irrealtà, Torino, Bollati Boringhieri, 2011.
} 
Ci si accorge così, dopo averne messa in discussione l'aproblematicità, di quale sia stata l'esclusione operata da tale sguardo: l'ontologia. Ai testi, cioè, non è mai stato chiesto di spiegare in quali mondi sia possibile dire la verità, né più in generale quali siano le caratteristiche della realtà che essi vorrebbero raccontare. Se tuttavia poniamo queste domande, immediatamente ci accorgiamo dell'esistenza di una seconda esclusione, correlata alla prima. È quella che ha colpito le preoccupazioni religiose presenti in molte opere contemporanee. La loro esistenza infatti, sebbene sia stata notata in più di una occasione, ${ }^{4}$ non ha mai portato ad alcuna modifica o ripensamento dell'interpretazione complessiva del periodo, a partire da quella del problema della verità. Non sembra tuttavia sbagliato, non almeno in via ipotetica, immaginare che ciò possa al contrario rivelarsi importante quando, mutato lo sguardo, si provi a capire quanto frequenti esse siano, e se abbiano un qualche legame con $i$ "ritorni" a realtà e verità del contemporaneo. Detto altrimenti, il fatto che il narratore di Campo del sangue descriva il suo viaggio verso Auschwitz come 'una vera iniziazione', ${ }^{5}$ che una delle saghe più famose degli ultimi trent'anni, quella che Evangelisti dedica all'inquisitore Eymerich, si apra con una citazione del corpus hermeticum, che cosa ci dicono sul contemporaneo?

Che valga la pena tentare di rispondere a queste domande, prima ancora che nel confronto con i testi, sembra venir confermato dalla rinnovata importanza che il credere, in una delle sue forme, ricopre nella vita delle società occidentali. Si tratterebbe di una rilegittimazione della fede che, secondo Gianni Vattimo, in Italia si coniuga con la fine del ruolo politico della Chiesa Cattolica, con quello del papato di Wojtyta e con la fine delle speranze di rinnovamento che, nel Novecento appunto, erano confluite nel marxismo. 'Il fatto è', scrive Vattimo, che con 'la "fine della modernità"' sembrano venir meno anche tutte "le ragioni filosofiche per essere atei, o comunque per rifiutare la religione'. ${ }^{6}$ Se dunque al di là delle diverse interpretazioni si può affermare che in Occidente e nella sua cultura sia rinnovato l'interesse per tutto ciò che ha a che fare con lo spirito, è possibile che di questo si trovi traccia anche nella letteratura. 0 , per meglio dire, che la letteratura partecipi, attivamente e in qualche modo, a queste stesse trasformazioni. Altrettanto importante è, per l'ipotesi che qui si formula, il fatto che studi all'incrocio tra letteratura e religione, e più precisamente tra forme contemporanee del credere e letteratura, non solo sono già stati tentati per quella nordamericana - si deve qui fare almeno il nome di John McClure - ma sono anche stati capaci di mostrare quale peso abbia avuto il religioso nel definirsi della produzione di molti autori.

Da queste premesse, e dal desiderio di provare a rispondere alle domande di cui abbiamo detto, nasce l'idea del convegno che ha ispirato i contributi raccolti nella sezione tematica di questo volume. La proposta che è stata fatta agli autori è stata cioè quella di uno spazio in cui parlare di letteratura e religione, lasciando il concetto il più aperto possibile ma richiamando al contempo le più recenti trasformazioni in materia. Su queste ultime è intervenuto Enzo Pace, con un saggio intitolato 'Deus ex machina: forme contemporanee del credere nel relativo', in cui a partire dai classici del pensiero sociologico si affronta il dibattito sociologico contemporaneo dando particolare risalto al rapporto tra religione e capitalismo; a questo testo si rimanda per una più precisa definizione delle trasformazioni a cui abbiamo accennato.

\footnotetext{
${ }^{4}$ Donnarumma per esempio nota l'importanza del modello fornito da Don Diana per il Saviano di Gomorra (Donnarumma, Ipermodernità, cit.; Simonetti indica la presenza di una rinascita mistica nella Vita oscena di Aldo Nove: 'C'è molto Petrolio [...] di sicuro nella Vita oscena di Aldo Nove: nell'io che si svuota attraverso la degradazione sessuale, e nella visione mistica di una seconda nascita' (G. Simonetti, La letteratura circostante, cit., pp. 263-264).

${ }^{5}$ E. Affinati, Campo del sangue, Milano, Mondadori, 1997, p. 31.

${ }^{6} \mathrm{G}$. Vattimo, Credere di credere, Milano, Garzanti, 1996, pp. 17-18.
} 
Alla poesia è invece dedicato il contributo di Michele Bordoni, intitolato 'Darti voce è incontrarti nelle cose'. Il canto di ritorno di Filippo Davoli tra religione e lirica. Qui la produzione di Davoli viene letta attraverso la lente del simbolo, e come caso di studio capace di mostrare quali siano i punti di contatto e le analogie tra ritorno del religioso e ritorno della lirica. Bordoni persegue il suo obbiettivo facendo riferimento da un lato alla riflessione di Lukács e Benjamin, e dall'altro a quella di Beck e Vattimo: benché, insomma, la poesia non sia stata all'origine delle domande che fin qui ci siamo posti, nate appunto dal confronto con la narrativa, l'intervento di Bordoni suggerisce che a quest'area si debba guardare con interesse.

Nell'articolo che Monica Jansen dedica al problema della ricezione di Sul concetto di volto nel Figlio di Dio e intitolato "Quando lo "scandalo" diventa provocazione. Il postsecolare e il teatro iconoclasta di Romeo Castellucci', viene esaminata la relazione, problematica, tra sfera pubblica e opera, nei casi in cui quest'ultima affronti temi religiosi. Più di preciso, Jansen studia le reazioni e le proteste che hanno accompagnato la messa in scena dello spettacolo di Castellucci, facendo ricorso a categorie provenienti dalla riflessione, tra gli altri, di Habermas e di Latour.

Marco Zonch, in un articolo intitolato 'Scritture postsecolari. Ipotesi su verità e spiritualità nella narrativa italiana contemporanea', attraverso alcuni esempi tratti dalla produzione di Emmanuele Trevi, Antonio Moresco e Aldo Nove tenta di delineare i tratti generali di un gruppo di opere che propone di chiamare 'scritture postsecolari'. La categoria vorrebbe provare a rendere conto, nel complesso, delle caratteristiche di quelle opere contemporanee che assegnano un ruolo centrale alla spiritualità. Viene definita facendo ricorso a strumenti tratti dalla riflessione di Michel Foucault e ad alcuni dei risultati della sociologia della religione contemporanea.

Si coglie infine l'occasione per ringraziare Incontri e le sue due caporedattrici, Inge Lanslots e Natalie Dupré, non solo per l'ospitalità accordata agli articoli qui contenuti, ma anche per la cordialità con la quale ne hanno guidato la pubblicazione.

\section{Marco Zonch}

Facoltà di Neofilologia - Dipartimento di Italianistica

Università di Varsavia

ul. Oboźna 8

00-332, Varsavia (Polonia)

marco.zonch@gmail.com 